Junge Phlebologie

\title{
Phlebo Start-Up: Erster Probedurchlauf mit Erfolg!
}

Im Rahmen der 60. Jahrestagung in Bielefeld Ende September 2018 startete erstmals für den studentischen Nachwuchs ein „Handson Phlebo Start-Up Workshop“.

Ausgerichtet ist dieser praktisch orientierte Kurs an die Nachwuchsgeneration, an Studenten in klinischen Semestern und angehende Ärzte im praktischen Jahr. Teilgenommen haben Studenten/innen aus den 4.-12. Semestern aus ganz Deutschland. Insgesamt konnte der Kurs beim ersten Durchlauf bereits 14 Anmeldungen vorweisen!

Nach der persönlichen Begrüßung durch den Kongresspräsidenten PD. Dr. Helger Stege ging es gleich zur Sache.

Im Hands-on Kurs wurden grundlegende phlebologische Themenkomplexe wie Kompressionstherapie, Duplexsonografie und Sklerotherapie vorgestellt. Hierzu wurde den Teilnehmern eine duplexsonografische Untersuchung am Patienten demonstriert, und die Kompressionssonografie mit anatomischen Grundlagen der Phlebologie vermittelt. Zusätzlich wurden Knotentechniken sowie unterschiedliche Nahttechniken am Modell gelehrt. Insbesondere die Herstellung des Polidocanolschaumes und das Üben am „Sklerodummy“ machte den Workshop Teilnehmern viel Spaß. Zukünftig wird es ebenfalls eine Station zum Thema endoluminale Techniken am Beispiel der endoluminalen Lasertherapie in Seldingertechnik geben.

Zusätzlich wurden die studentischen Teilnehmer eingeladen den Crash Kurs am Mittwochvormittag zu besuchen, um die wichtigsten Grundlagen der Phlebologie zu erlernen und zu vertiefen. Spezialisiert werden konnte das Wissen am Donnerstagvormittag im Rahmen des Weiterbildungsforums durch Vorträge der jungen Phlebologen. Abschließend konnten Kontakte geknüpft wer-

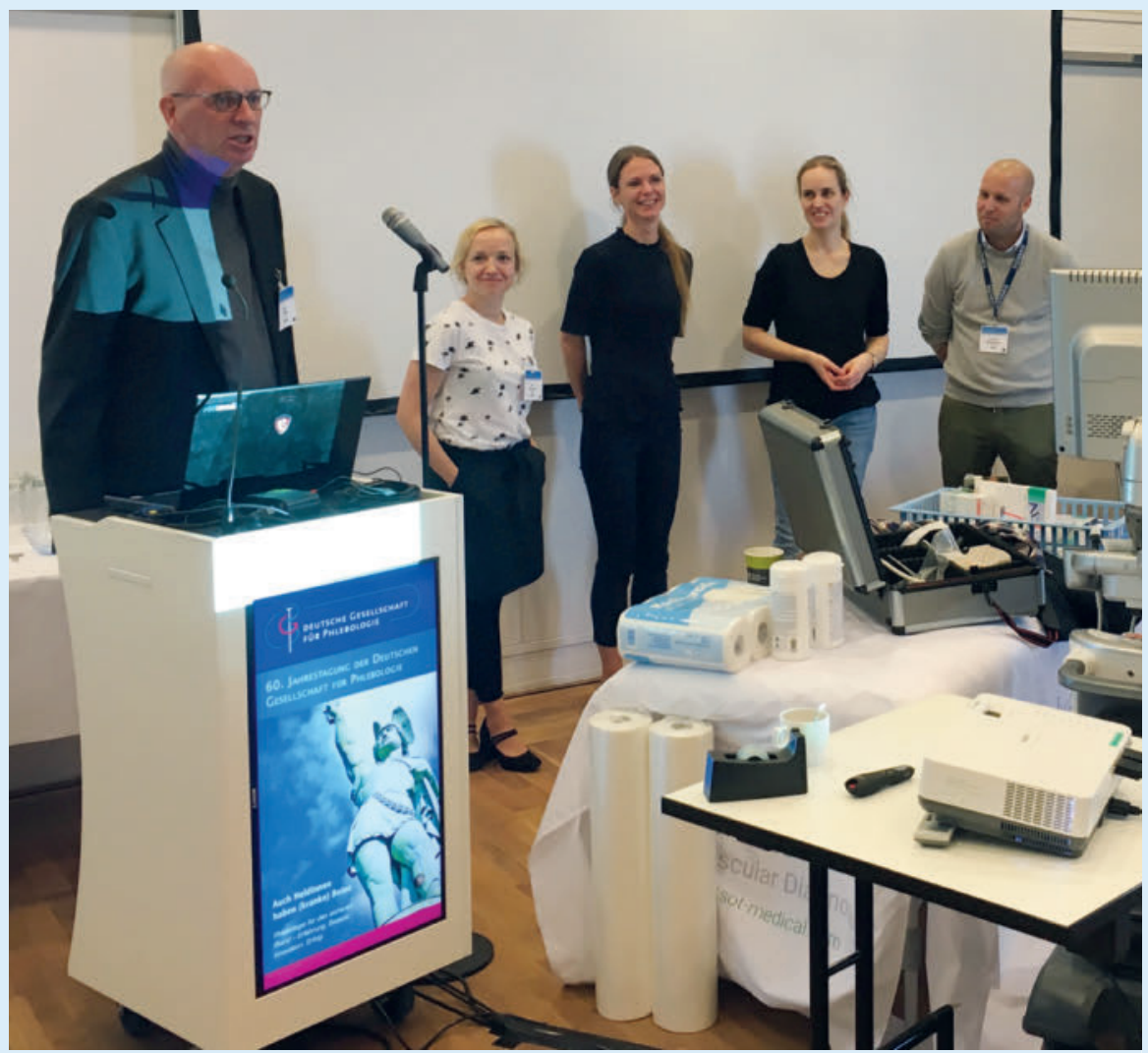

- Abb. 1 Begrüßung durch den Kongresspräsidenten und die Dozenten. V.I. n. r: Kongresspräsident Priv.-Doz. Dr. med. H. Stege, Detmold, Dr. med. N. Devereux, Hamburg, Dr. med. J. Woitalla-Bruning, Hamburg, Dr. med. A. L. Recke, Lübeck, Dr. dr. med. D. Mühlberger, Bochum.

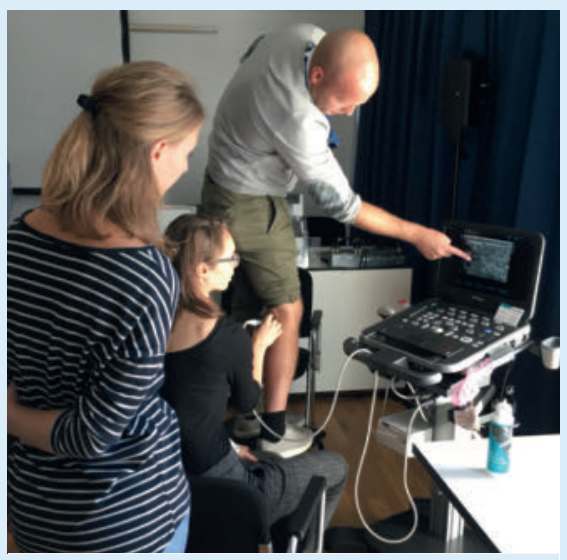

- Abb. 2 Duplexsonografie der Beinvenen durch die Kursteilnehmer.

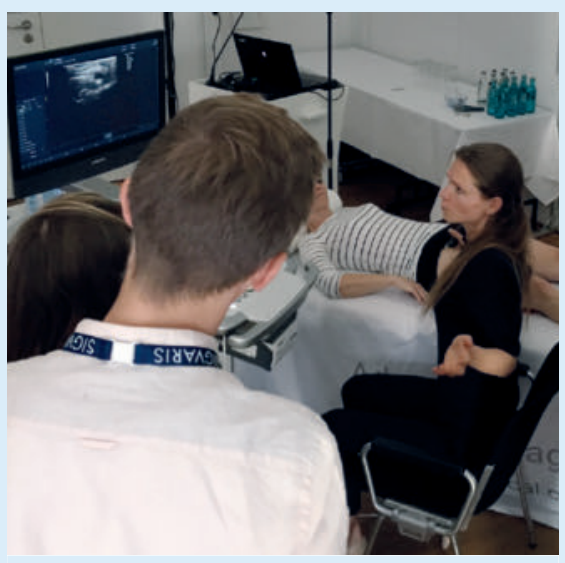

Abb. 3 Demonstration der Kompressionssonografie. 


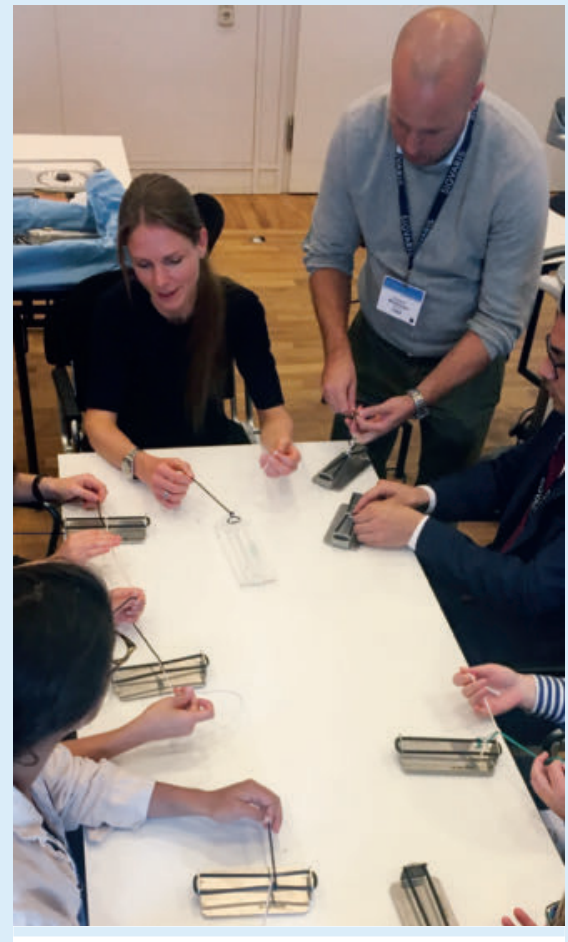

- Abb. 4 Erlernen von Knoten- und Nahttechniken am Modell.

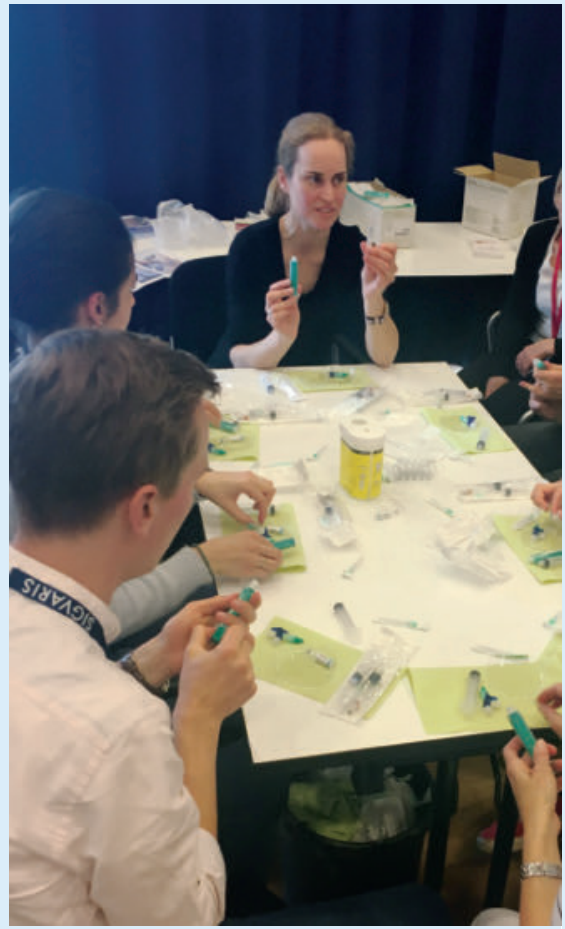

- Abb. 5 Herstellung von Sklerosierungsschaum. den und fachliche Gespräche am Abend bei der Phlebo-Party im MOVIE geführt werden. Für das leibliche Wohl sowie für die Übernachtung wurde gesorgt. Die Anreise erfolgte auf eigene Faust. Finanziell unterstützt und organisiert wurde der Workshop durch die Deutsche Gesellschaft für Phlebologie sowie von Sigvaris.

Aufgrund des erfolgreichen Starts und des positiven Feedbacks freuen wir uns schon jetzt bekannt geben zu können, dass auch im kommenden Jahr während der Kongresstagung in Münster vom 18.-21. September 2019 (voraussichtlich am Mittwochnachmittag) erneut ein studentischer Workshop angeboten wird. Bei Interesse freuen wir uns über frühzeitige Anmeldungen im Sekretariat der deutschen Gesellschaft für Phlebologie bei Frau Pielhau, E-Mail: sekretariat@ phlebology. de oder telefonisch +49(0)2282871-6959.

Interessenkonflikt

Reisekostenunterstützung zu Kongressen durch die Firma Bauerfeind.

Kongressshonorar durch die Firma Sigvaris

Dr. Jasmin Woitalla-Bruning

Tabea Krankenhaus

Zentrum für Venen- und Dermatochirurgie

Hauttumorzentrum Hamburg am

Krankenhaus Tabea

Kösterbergstrasse 32, 22587 Hamburg 